\title{
Status of Surgical Treatment of Biliary Tract Cancer
}

\author{
Shin Ishihara a, b Shuichi Miyakawa ${ }^{a, b}$ Tadahiro Takadac Ken Takasaki ${ }^{d}$ \\ Yuji Nimura ${ }^{\mathrm{e}}$ Masao Tanaka ${ }^{\mathrm{f}}$ Masaru Miyazakig ${ }^{\text {Takukazu Nagakawa }}{ }^{\mathrm{h}}$ \\ Masato Kayahara ${ }^{i}$ Akihiko Horiguchi ${ }^{a, b}$ \\ a Department of Biliary Pancreatic Surgery, Fujita Health University, Toyoake; ${ }^{b}$ Registration Secretariat, \\ Biliary Tract Cancer, Toyoake; 'Department of Surgery, Teikyo University, Tokyo; d Department of Surgery,

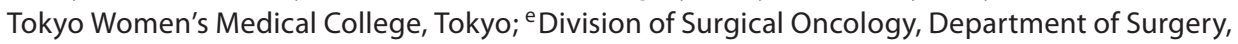 \\ Nagoya University Graduate School of Medicine, Nagoya; ${ }^{f}$ Department of Surgery and Oncology, \\ Graduate School of Medical Science, Kyusyu University, Fukuoka; ${ }^{9}$ Department of General Surgery, \\ Graduate School of Medicine, Chiba; ' $F u k u n o$ Hospital, Nanto, and 'Department of Gastroenterologic Surgery, \\ Division of Cancer Medicine, Graduate School of Medical Science, Kanazawa University, Kanazawa, Japan
}

\section{Key Words}

Biliary tract carcinoma - Bile duct carcinoma, hilar, middle, distal $\cdot$ Gallbladder carcinoma $\cdot$ Papilla of Vater carcinoma Biliary surgery, 5 -year survival · Disease stage

\begin{abstract}
Complete surgical resection of biliary tract carcinoma remains the best treatment. The Japanese Society of Biliary Surgery has organized a registry project and established a classification of biliary tract carcinoma. We report here the status of biliary surgery in Japan. For hilar bile duct carcinoma, major hepatectomy is needed to increase the resection rate, and total caudate lobectomy is required for curative resection. The 5 -year survival rate was 39.1\%. Middle and distal bile duct carcinomas were treated with pancreatoduodenectomy (PD) or pylorus-preserving PD (PPPD) or bile duct resection alone. The 5-year survival rate was $44.0 \%$. The treatment of gallbladder carcinoma with pT1 lesions is cholecystectomy. The treatment of pT2 lesions is extended cholecystectomy or various hepatectomy with or without extrahepatic bile duct resection along with lymphadenectomy. Treatment of pT3 and pT4 lesions includes hepatectomy with or without bile duct resection, combined with vascular re-
\end{abstract}

section, extended lymphadenectomy, and autonomic nerve dissection. Several groups in Japan have performed hepatopancreatoduodenectomy. The 5-year survival rate of pT1, pT2, pT3, and pT4 were 93.7, 65.1, 27.3, and 13.8\%. PD or PPPD is the standard operation for carcinoma of the papilla of Vater. The 5-year survival rate was $57.5 \%$.

Copyright $\odot 2007$ S. Karger AG, Basel

\section{Introduction}

Complete surgical resection of biliary tract carcinoma remains the best treatment for long-term survival. In Japan, the Japanese Society of Biliary Surgery (JSBS) is organized into 225 institutions. It performs registration of biliary tract carcinomas as one of its projects. In this project, the society has established guidelines for the treatment of cancer of the biliary tract based on the extent of involvement at each site. A total of 3,518 cases of biliary tract carcinoma were registered between 1998 and 2002; the site of carcinoma was the bile duct in 1,669, the gallbladder in 1,345, and the papilla of Vater in 504 cases. These cases were analyzed with regard to patient survival. We report the status of biliary surgery in Japan.

\section{KARGER \\ Fax +41613061234 E-Mail karger@karger.ch} www.karger.com (c) 2007 S. Karger AG, Basel $0253-4886 / 07 / 0242-0131 \$ 23.50 / 0$

Accessible online at: www.karger.com/dsu
Shin Ishihara

1-98 Dengakugakubo, Kutsukake-cho

Toyoake, Aichi 470-1192 (Japan)

Tel. +81 56293 9246, Fax +81 562930109

E-Mail ishin@fujita-hu.ac.jp 


\section{Classification of Biliary Tract Carcinoma}

As noted above, the JSBS established guidelines for the treatment of cancer of the biliary tract based on the extent of involvement at each site, according to the Classification of Biliary Tract Carcinoma currently used in Japan, and the 2nd English edition was published in 2004 [1]. The guidelines promoted in Japan for the treatment of biliary tract carcinoma are divided into three anatomical regions: the biliary duct, gallbladder, and papilla of Vater.

\section{Bile Duct Carcinoma}

\section{Staging}

Extrahepatic carcinomas have been subdivided into proximal or hilar, middle, and distal subgroups. The histological extent of tumor invasion around the bile duct ( $t$ category) in the classification of biliary tract carcinoma of JSBS is defined as the degree of tumor extension. According to the currently used Japanese classification of tumor invasion into the bile duct wall, serosal invasion is histologically divided into 5 stages, $\mathrm{m}$, fm, ss, se, and si, in anatomical fashion. Furthermore, various types of direct invasion of the carcinoma into four structures present around the bile duct, i.e., invasion of the hepatic parenchyma (hinf), pancreatic parenchyma (panc), portal venous system (p), and arterial system (a), are graded from 0 to 3. Nodal involvement of carcinoma is classified into four groups. The stages of biliary tract carcinoma of JSBS are classified into five groups [1].

\section{Hilar Bile Duct Carcinoma}

Long-term survival with hilar bile duct carcinoma depends critically on complete resection with negative margins. The resection rate increases with the performance of major hepatectomy, and the likelihood of negative margins by performing it rises in hilar bile duct carcinoma. In addition, total caudate lobectomy is required for curative resection since caudate branches join the hilar bile duct $[2,3]$. However, the risk of developing hepatic failure as a postoperative complication increases when major hepatectomy is performed. Therefore, when major hepatectomy is planned, portal vein embolization (PVE) is performed in many Japanese institutions. The main purpose of PVE is to induce compensatory hypertrophy of the future remnant liver and thus minimize postoperative liver dysfunction. In the recent literature from high-volume centers in Japan [4-11], the mortality
Table 1. Surgical procedure for hilar bile duct carcinoma

\begin{tabular}{lclll}
\hline Type of hepatectomy & Cases & $\begin{array}{l}\text { With } \\
\text { PD }\end{array}$ & $\begin{array}{l}\text { With } \\
\text { PV }\end{array}$ & $\begin{array}{l}\text { With } \\
\text { HA }\end{array}$ \\
\hline Left hepatectomy & 54 & 2 & 2 & 1 \\
Extended left hepatectomy & 66 & 9 & 6 & 3 \\
Left trisegmentectomy & 10 & 1 & 2 & \\
Right hepatectomy & 35 & 8 & 1 & \\
Extended right hepatectomy & 69 & 9 & 4 & \\
Right trisegmentectomy & 18 & 4 & 2 & \\
Central bisegmentectomy & 3 & & & \\
\hline Total & 255 & 29 & 17 & 8
\end{tabular}

$\mathrm{PD}=$ Pancreatoduodenectomy; $\mathrm{PV}=$ resection and construction of the portal vein; $\mathrm{HA}=$ resection and construction of the hepatic artery.

rate ranged from 0 to $12 \%$. The overall 3 - and 5 -year survival rates ranged from 26 to $54.8 \%$ and 23 to $40 \%$, respectively. The proportion of stage IVa (5th edition of UICC) or over stage III (6th edition of UICC) ranged from 41 to $72 \%$. A total of 255 cases of hilar bile duct carcinoma subjected to major hepatectomy were registered in the JSBS between 1998 and 2002 (table 1). The surgical procedure was left hepatectomy in 54 patients, extended left hepatectomy in 66 patients, left trisegmentectomy in 10 patients, right hepatectomy in 35 patients, extended right hepatectomy in 69 patients, right trisegmentectomy in 18 patients, and central bisegmentectomy in 3 patients. Bloc hepatic resection with pancreatoduodenectomy (HPD) was performed in 29 patients. Portal vein resection and reconstruction was performed in $167 \mathrm{pa}-$ tients. Resection of the right or left hepatic artery and reconstruction was performed in 78 patients. The overall the 1-, 2-, 3-, and 5-year survival rates of patients who underwent major hepatectomy were 77.3, 55.9, 46.7, and $39.1 \%$, respectively. Patients were grouped according to the Japanese classification as stage I $(\mathrm{n}=21,8.2 \%)$, stage II $(\mathrm{n}=45,17.6 \%)$, stage III $(\mathrm{n}=66,25.9 \%)$, stage IVa $(\mathrm{n}=86,33.7 \%)$, or stage IVb $(\mathrm{n}=37,14.5 \%)$. The 5-year survival rate was $90.0 \%$ in stage I patients, $57.7 \%$ in stage II, $46.2 \%$ in stage III, $29.9 \%$ in stage IVa, and $17.0 \%$ in stage IVb patients (fig. 1). Compared with high-volume centers in Japan, the overall survival rate of the JSBS is good. This difference in survival rate is attributable to the higher percentage of cases of advanced cancer among those undergoing surgery at high-volume centers in Japan where aggressive surgery is often performed for advanced cancer. 


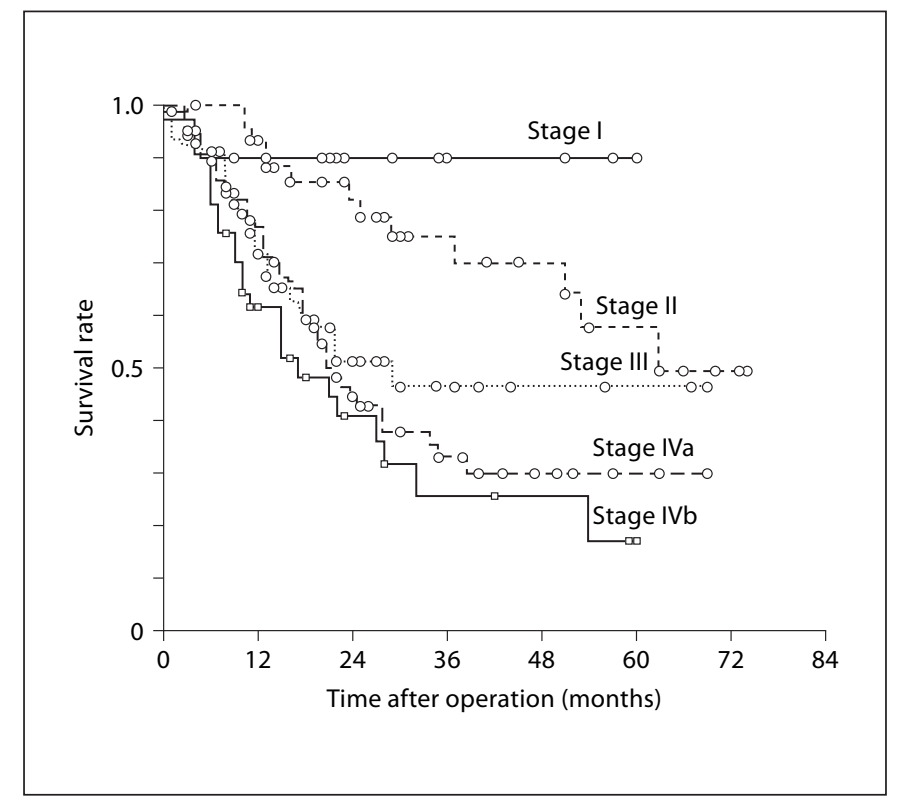

Fig. 1. Survival rates of hilar bile duct carcinoma according to stage.

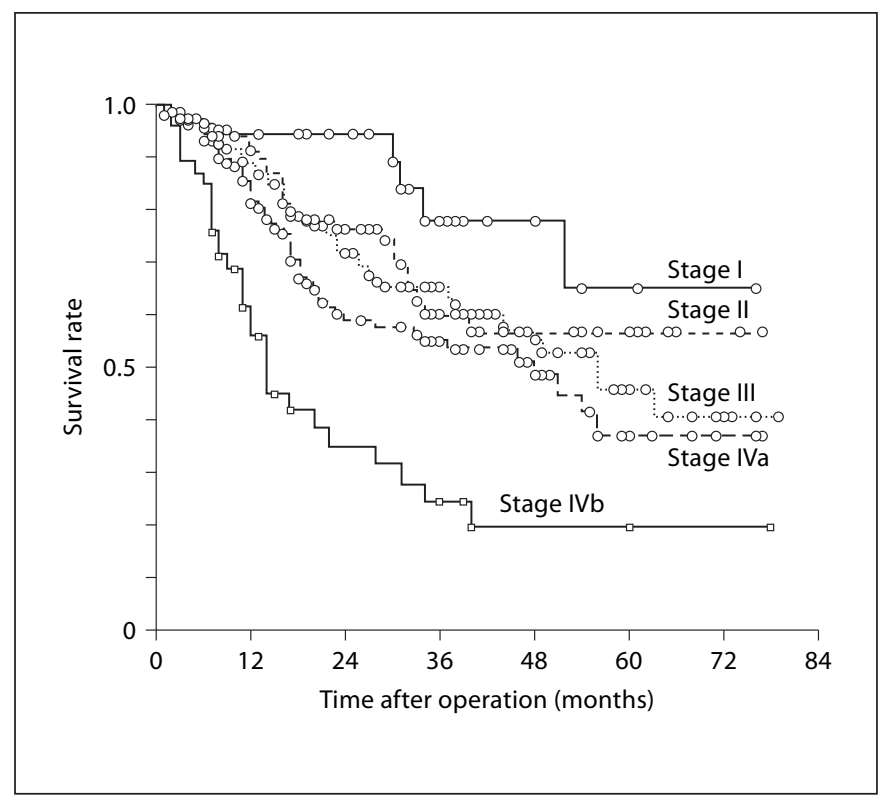

Fig. 2. Survival rates of middle and distal bile duct carcinoma according to stage.
Table 2. Studies of middle and distal bile duct carcinoma

\begin{tabular}{llllll}
\hline Reference & Year & $\begin{array}{l}\text { Location } \\
\text { of tumor }\end{array}$ & Patients & $\begin{array}{l}\text { 3-Year } \\
\text { survival, \% }\end{array}$ & $\begin{array}{l}\text { 5-Year } \\
\text { survival, \% }\end{array}$ \\
\hline Yamaguchi et al. [12] & 1997 & Distal & 11 & 38 & 19 \\
Yamaguchi et al. [12] & 1997 & Middle & 11 & 33 & 11 \\
Kayahara et al. [13] & 1999 & Middle and distal & 50 & 47 & 35 \\
Suzuki et al. [14] & 2000 & Middle and distal & 99 & 50 & 37.4 \\
Sasaki et al. [15] & 2001 & Middle and distal & 59 & 42.6 & 33.6 \\
Yoshida et al. [16] & 2002 & Distal & 27 & 37 & 37 \\
Sakamoto et al. [17] & 2005 & Middle and distal & 55 & 52 & 24 \\
\hline
\end{tabular}

\section{Middle and Distal Bile Duct Carcinoma}

Middle and distal bile duct carcinoma is treated with pancreatoduodenectomy (PD) or pylorus-preserving $\mathrm{PD}$ (PPPD) or bile duct resection alone. In Japan, some surgeons have advocated complete removal of the primary bile duct cancer with connective tissue clearance, including lymph nodes and neural plexus dissection. Recent results reported from high-volume centers in Japan are summarized (table 2) [12-17]. The overall 3- and 5-year survival rates ranged from 33 to $52 \%$ and 11 to $37.4 \%$, respectively. 427 patients with middle and duct carcinoma, excluding those with insufficient data, who underwent PD or PPPD were registered in the JSBS between 1998 and 2002. The overall 1-, 2-, 3-, and 5-year survival rates were $83.5,66.6,57.9$, and $44.0 \%$, respectively. Patients were grouped according to the Japanese classification as stage I $(\mathrm{n}=36,8.4 \%)$, stage II $(\mathrm{n}=82,19.2 \%)$, stage III $(\mathrm{n}=134$, $31.4 \%)$, stage IVa $(\mathrm{n}=130,30.4 \%)$, or stage IVb $(\mathrm{n}=45$, $10.5 \%)$. The 5 -year survival rates were $64.8 \%$ in stage I patients, $56.9 \%$ in stage II, $45.6 \%$ in stage III, $36.8 \%$ in stage IVa, and $19.5 \%$ in stage IVb (fig. 2).

\section{Gallbladder Carcinoma}

\section{Staging}

The histological extent of tumor invasion around the gallbladder (t category) in the classification of gallbladder carcinoma of the JSBS is defined as the degree of tumor extension. According to the currently employed Japanese 


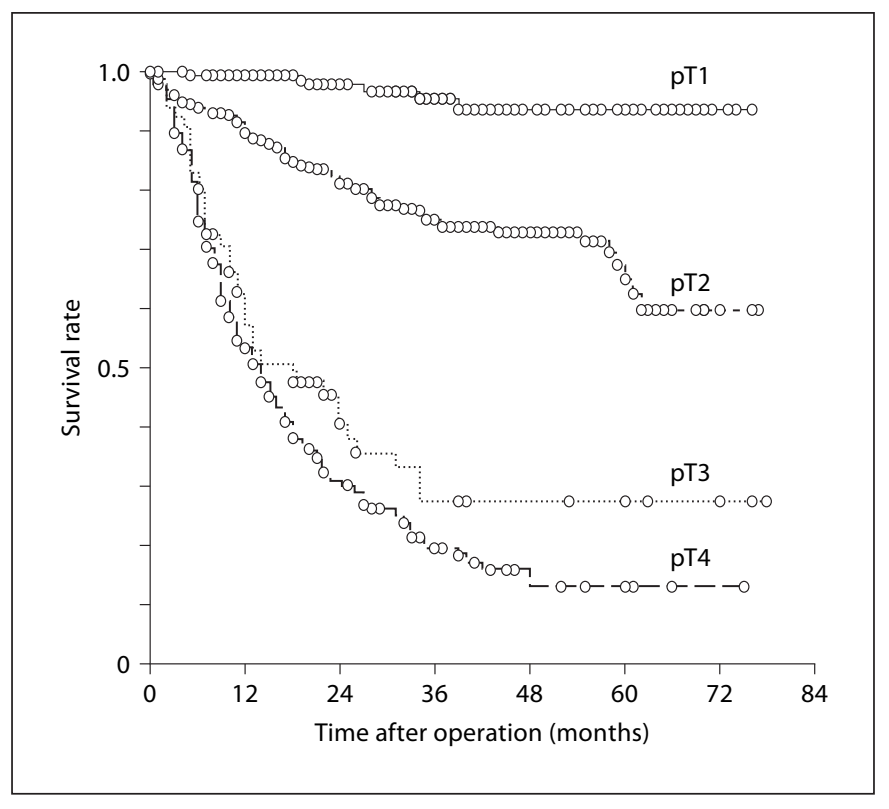

Fig. 3. Survival rates of gallbladder carcinoma according to depth of invasion.

classification of tumor invasion into the bile duct wall, serosal invasion is histologically classified into five stages, $\mathrm{m}, \mathrm{mp}$, ss, se, and si, in accordance with the anatomical structure. Furthermore, various types of direct invasion of carcinoma into four structures present around the bile duct are distinguished, i.e., invasion of the hepatic parenchyma (hinf), hepatoduodenal ligament (binf), portal venous system (p), and arterial system (a), which are graded from 0 to 3 . Nodal involvement of gallbladder carcinoma is classified into four groups. The stages of biliary tract carcinoma of the JSBS are classified into five groups [1].

\section{Surgery}

Most surgeons agree that $\mathrm{pT} 1$ tumors are effectively treated with cholecystectomy. The 1-, 2-, 3-, and 5-year survival rates of $\mathrm{pT} 1$ patients $(\mathrm{n}=160)$ registered with the JSBS were 99.4, 97.8, 95.4, and 93.7\%, respectively (fig. 3). pT2 tumors often exhibit lymph node metastases. $30 \%$ (93/306) of the T2 tumors registered with JSBS were associated with lymph node metastases. Lymph node dissection is thus necessary for pT2 gallbladder carcinoma. Extended cholecystectomy or various types of hepatectomy with or without extrahepatic bile duct resection have been performed for $\mathrm{pT} 2$ patients in Japan. The range of liver resection required as part of radical surgery is still controversial. The 1-, 2-, 3-, and 5-year survival rates of

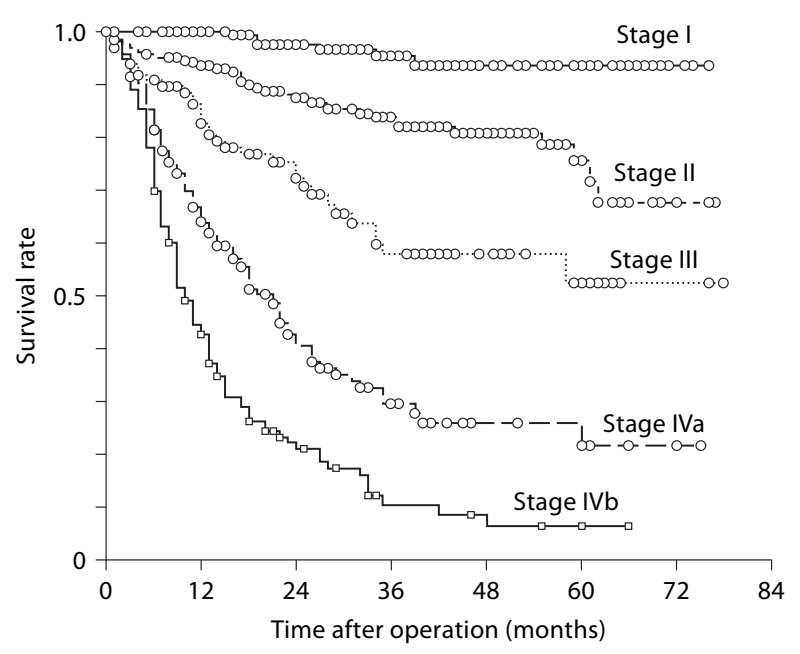

Fig. 4. Survival rates of gallbladder carcinoma according to stage.

T2 patients $(\mathrm{n}=306)$ registered with the JSBS were 89.8 , $81.1,75.2$, and $65.1 \%$, respectively (fig. 3). For pT3 and pT4 tumors, the surgical procedures currently used in Japan include various types of hepatectomy with or without bile duct resection, combined vascular resection, extended lymphadenectomy, and autonomic nerve dissection. Several surgical groups in Japan have performed HPD for locally advanced gallbladder carcinoma. The usefulness of extrahepatic bile duct resection as part of radical surgery for advanced gallbladder carcinoma is also still controversial, particularly when there is no apparent extrahepatic bile duct involvement. The 1-, 2-, 3-, and 5-year survival rates of $\mathrm{pT} 3$ patients $(\mathrm{n}=66)$ registered with the JSBS were 57.7, 41.0, 27.3, and 27.3\%, while those for pT4 patients $(n=228)$ were $53.6,30.3,19.5$, and $13.8 \%$, respectively (fig. 3).

760 patients with gallbladder carcinoma, excluding those with insufficient data, who underwent resection were registered in the JSBS between 1998 and 2002. The overall 1-, 2-, 3-, and 5-year survival rates were 78.2, 66.3, 58.9 , and $52.6 \%$, respectively. There are two papers concerning large numbers in Japan from different periods. The 5 -year survival rate of 1,686 patients with resection between 1979 and 1988 was $30.1 \%$ [18], while that of 3,249 patients with resection between 1988 and 1998 (JSBS) was $42 \%$ [19]. Patients were grouped according to the Japanese classification as stage I $(n=160,8.4 \%)$, stage II 


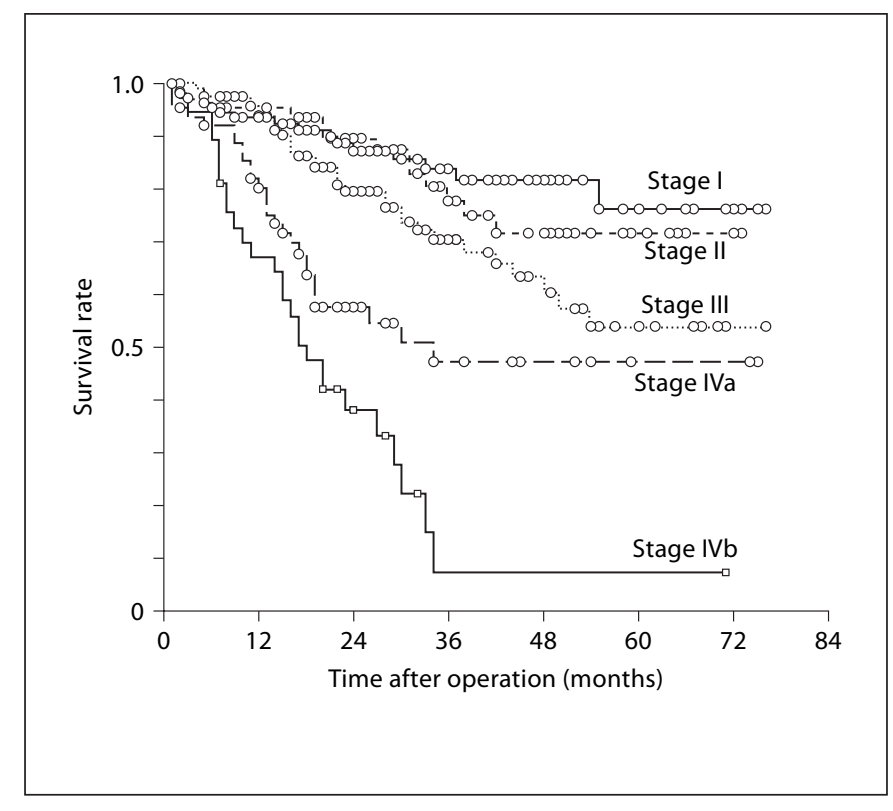

Fig. 5. Survival rates of carcinoma of the papilla of Vater according to stage.

Table 3. Studies of carcinoma of the papilla of Vater

\begin{tabular}{lllll}
\hline Reference & Year & Patients & $\begin{array}{l}\text { 3-Year } \\
\text { survival, \% }\end{array}$ & $\begin{array}{l}\text { 5-Year } \\
\text { survival, \% }\end{array}$ \\
\hline Kawarada et al. [20] & 1993 & 89 & 63.6 & 57.4 \\
Nakao et al. [21] & 1994 & 26 & 57.7 & 52.3 \\
Shirai et al. [22] & 1996 & 35 & 60 & \\
Kayahara et al. [23] & 1997 & 36 & 66 & 56 \\
Tanaka et al. [24] & 2002 & 16 & 58.9 & 55 \\
\hline
\end{tabular}

$(\mathrm{n}=208,19.2 \%)$, stage III $(\mathrm{n}=98,31.4 \%)$, stage IVa $(\mathrm{n}=$ $152,30.4 \%)$, or stage IVb $(\mathrm{n}=142,10.5 \%)$. The 5 -year survival rate was $93.6 \%$ in stage I patients, $80.8 \%$ in stage II, $52.6 \%$ in stage III, $21.5 \%$ in stage IVa, and $6.5 \%$ in stage $\mathrm{IVb}$ (fig. 4).

\section{Carcinoma of the Papilla of Vater}

\section{Staging}

The histological extent of tumor invasion around the papilla of Vater (t category) in the classification of gallbladder carcinoma of the JSBS is defined as the degree of tumor extension. Various types of histologically direct invasion of the carcinoma into two structures present around the papilla of Vater, i.e. the pancreatic parenchy$\mathrm{ma}$ (panc) and duodenum (du), are graded from 0 to 3. Nodal involvement of gallbladder carcinoma is classified into four groups. The stages of biliary tract carcinoma of JSBS are classified into five groups [1].

\section{Surgery}

In most series, the resectability rate is higher than for other malignant tumors of the periampullary region. PD or PPPD is the standard operation for carcinoma of the papilla of Vater. Recent reports from high-volume centers in Japan are summarized (table 3) [20-24]. The overall 3 - and 5-year survival rates ranged from 55 to $66 \%$ and from 40 to $60 \%$, respectively. 404 patients with carcinoma of papilla of Vater, excluding those with insufficient data, who underwent PD or PPPD were registered in the JSBS between 1998 and 2002. The overall 1-, 2-, 3-, and 5 -year survival rates were $89.3,75.6,66.0$, and $57.5 \%$, respectively. Patients were grouped according to the Japanese classification as stage I $(\mathrm{n}=112,27.7 \%)$, stage II $(\mathrm{n}=$ $65,16.1 \%)$, stage III $(\mathrm{n}=126,31.2 \%)$, stage IVa $(\mathrm{n}=64$, $15.8 \%)$, or stage IVb $(n=37,9.2 \%)$. The 5 -year survival rates were $76.3 \%$ in stage I patients, $71.7 \%$ in stage II, $54.0 \%$ in stage III, $47.2 \%$ in stage IVa, and $7.4 \%$ in stage $\mathrm{IVb}$ (fig. 5).

\section{Conclusion}

We report the status of biliary surgery in Japan. Hilar bile duct carcinoma is one of the diseases on which Japanese biliary tract surgeons place particular emphasis. PVE performed during major hepatectomy and total caudate lobectomy have contributed to improving the outcome of treatment of hilar bile duct carcinoma. Middle and distal bile duct carcinomas are treated with PD or PPPD or bile duct resection alone. The treatment of gallbladder carcinoma with pT1 lesions is cholecystectomy. The treatment of pT2 lesions is extended cholecystectomy or various hepatectomy with or without extrahepatic bile duct resection, and lymphadenectomy. The treatment of pT3 and pT4 lesions includes various types of hepatectomy with or without bile duct resection combined vascular resection, extended lymphadenectomy, and autonomic nerve dissection. The usefulness of resection of the extrahepatic bile duct and the range of liver resection of gallbladder carcinoma are still controversial. Several groups in Japan perform HPD for locally advanced gallbladder carcinoma. PD or PPPD is the standard operation for carcinoma of the papilla of Vater. 


\section{References}

1 Japanese Society of Biliary Surgery: Classification of Biliary Tract Carcinoma, second English edition. Tokyo, Kanehara, 2004.

-2 Mizumoto R, Suzuki H: Surgical anatomy of hepatic hilum with special reference to the caudate lobe. World J Surg 1988;12:2-10.

-3 Nimura Y, Hayakawa N, Kamiya J, et al: Hepatic segmentectomy with caudate lobe resection for bile duct carcinoma of the hepatic hilus. World J Surg 1990;14:535-544.

4 Miyazaki M, Ito H, Nakagawa K, et al: Parenchyma-preserving hepatectomy in surgical treatment of hilar cholangiocarcinoma. J Am Coll Surg 1999;189:575-583.

5 Kosuge T, Yamamoto J, Shimada K, et al: Improved surgery results for hilar cholangiocarcinoma. Ann Surg 1999;230:663-671.

6 Todoroki T, Kawamoto T, Koike N, et al: Radical resection of hilar bile duct carcinoma and predictors of survival. Br J Surg 2000; 87:306-313.

7 Tabata M, Kawarada Y, Yokoi H, et al: Surgical treatment for hilar cholangiocarcinoma. J Hepatobiliary Pancreat Surg 2000;7:148154.

$\checkmark 8$ Seyama Y, Kubota K, Sano K, et al: Longterm outcome of extended hemihepatectomy for hilar bile duct cancer with no mortality and high survival rate. Ann Surg 2003;238: 73-83.

-9 Kawasaki S, Imamura H, Kobayashi A, et al: Results of surgical resection for patients with hilar bile duct cancer: application of extended hepatectomy after biliary drainage and hemihepatic portal vein embolization. Ann Surg 2003;238:84-92.
10 Kondo S, Hirano S, Ambo Y, et al: Forty consecutive resections of hilar cholangiocarcinoma with no postoperative mortality and no positive ductal margins: results of a prospective study. Ann Surg 2004;240:95-101.

11 Nagino S, Kamiya J, Nishio H, et al: Two hundred forty consecutive portal vein embolizations before extended hepatectomy for biliary cancer: surgical outcome and longterm follow-up. Ann Surg 2006;243:364372.

12 Yamaguchi K, Chijiiwa K, Saiki S, et al: Carcinoma of extrahepatic bile duct: mode of spread and its prognostic implications. Hepatogastroenterology 1997;44:1256-1261.

13 Kayahara M, Nagakawa T, Ohta T, et al: Role of nodal involvement and the periductal soft-tissue margin in middle and distal bile duct cancer. Ann Surg 1999;229:76-83.

14 Suzuki M, Unno M, Oikawa M, et al: Surgical treatment and postoperative outcomes for middle and lower bile duct carcinoma in Japan-experience of a single institute. Hepatogastroenterology 2000;47:650-657.

15 Sasaki R, Takahashi M, Funato O, et al: Prognostic significance of lymph node involvement in middle and distal bile duct cancer. Surgery 2001;129:677-683.

16 Yoshida T, Matsumoto T, Sasaki A, et al: Prognostic factors after pancreatoduodenectomy with extended lymphadenectomy for distal bile duct cancer. Arch Surg 2002; 137:69-73.
17 Sakamoto Y, Kosuge T, Shimada K, et al: Prognostic factors of surgical resection in middle and distal bile duct cancer: an analysis of 55 patients concerning the significance of ductal and radical margins. Surgery 2005; 137:396-402.

18 Ogura Y, Mizumoto R, Isaji S, et al: Radical options for carcinoma of gallbladder: present status in Japan. World J Surg 1991;15:337343.

19 Nagakawa T, Kayahara M, Ikeda S, et al: Biliary tract cancer treatment: results from the biliary tract cancer statistics registry in Japan. J Hepatobiliary Pancreat Surg 2002;9: 569-575.

20 Kawarada Y, Takahashi K, Tabata M, et al: Surgical treatment for carcinoma of the papilla of Vater. J Hepatobiliary Pancreat Surg 1993;1:8-13.

21 Nakao A, Harada A, Nonami T, et al: Prognosis of cancer of the duodenal papilla of Vater in relation to clinicopathological tumor extension. Hepatogastroenterology 1994;41:650-657.

22 Shirai Y, Tsukada K, Ohtani T, et al: Carcinoma of the ampulla of Vater: is radical lymphadenectomy beneficial to patients with nodal disease? J Surg Oncol 1996;61: 190-194.

23 Kayahara M, Nagakawa T, Ohta T, et al: Surgical strategy for carcinoma of the papilla of Vater on basis of lymphatic spread and mode recurrence. Surgery 1997;121:611-617.

$\checkmark 24$ Tanaka S, Hirohashi K, Tanaka H, et al: Prognostic factors in patient with carcinoma of the papilla of Vater. Hepatogastroenterology 2002;49:1116-1119. 